\title{
IDENTIFICAÇÃO DE FONTES DE PRODUÇÃO DE SEDIMENTOS EM UMA BACIA HIDROGRÁFICA DE $\operatorname{ENCOSTA}^{(1)}$
}

\author{
Pablo Miguel $^{(2)}$, Ricardo Simão Diniz Dalmolin ${ }^{(3)}$, Fabrício de Araújo Pedron ${ }^{(4)}$, Jean \\ Michel Moura-Bueno( ${ }^{(5)}$ \& Tales Tiecher ${ }^{(6)}$
}

\begin{abstract}
RESUMO
O uso do solo fora de sua aptidão agrícola é o que norteia a degradação dos recursos naturais por meio das atividades antrópicas. As atividades agropecuárias estão entre as que mais perturbam o meio ambiente, expondo o solo à ação dos processos erosivos e acelerando a transferência de sedimentos aos corpos de água. Nos últimos anos, o método fingerprinting para identificação de fontes de sedimentos tem sido utilizado com sucesso no mundo, porém, trabalhos dessa natureza realizados no Brasil ainda estão em caráter incipiente. $O$ objetivo deste trabalho foi estimar a proporção de contribuição das principais fontes de produção de sedimentos de uma bacia hidrográfica de encosta, onde predominam solos rasos e agricultura menos intensiva no sistema familiar. Essa bacia está localizada na região central do Estado do Rio Grande do Sul, tendo por ocupação do solo predominante áreas de floresta nativa seguida das de campo nativo, capoeira, lavouras anuais e, em menor expressão, silvicultura (eucalipto) e área urbanizada. Para a identificação das fontes, foi utilizado o método fingerprinting, que compara os solos de diferentes fontes, e os sedimentos, que são encontrados em suspensão no canal de drenagem, usando elementos traçadores. As maiores contribuições na produção de sedimentos são provenientes da erosão superficial do solo. Para o primeiro período de monitoramento, foi identificada uma produção relativa de sedimentos de $60 \%$ das estradas, para o ponto T2; de $100 \%$ da malha, para o T3; e de $77 \%$ da malha, para o T4. Para o segundo período de monitoramento, as estradas contribuíram relativamente com $81 \%$ do sedimento em suspensão no ponto T2 e com $76 \%$, no T3. Já no ponto T4, a maior contribuição foi da malha com $82 \%$. $O$
\end{abstract}

(1) Recebido para publicação em 11 de abril de 2013 e aprovado em 11 de dezembro de 2013.

(2) Professor Auxiliar do Departamento de Solos, Faculdade de Agronomia Eliseu Maciel, Universidade Federal de Pelotas. Campus Universitário, s/n. Caixa Postal 354. CEP 96010-900 Pelotas (RS). E-mail: pablo.ufsm@gmail.com

(3) Professor Associado, Departamento de Solos, UFSM. Bolsista PQ CNPq. E-mail: dalmolin@ufsm.br

(4) Professor Adjunto, Departamento de Solos, UFSM. E-mail: fapedron@ufsm.br

(5) Mestrando em Ciência do Solo, Departamento de Solos, UFSM. Bolsista CAPES. E-mail: bueno.jean1@gmail.com

(6) Doutorando em Ciência do Solo, Departamento de Solos, Universidade Federal de Santa Maria, UFSM. Campus Universitário. Av. Roraima, 1000, Bairro Camobi. CEP 97105-900 Santa Maria (RS). Bolsista CAPES. E-mail: tales.t@hotmail.com 
método se apresentou sensível em detectar mudanças na contribuição de cada fonte em razão das características apresentadas por cada uma delas, para o tipo de bacia hidrográfica estudada.

\title{
Termos de indexação: fingerprinting, elementos traçadores, erosão hídrica, áreas
} de encosta.

\section{SUMMARY: IDENTIFYING SOURCES OF SEDIMENT PRODUCTION IN A HILLSIDE CATCHMENT AREA}

\begin{abstract}
Land use outside of its agricultural suitability is the main cause of degradation of natural resources due to anthropogenic activities. Agricultural activities are among those that most disturb the environment, exposing the soil to the action of water erosion and accelerating the transfer of sediments into bodies of water. In recent years, the fingerprinting method for identifying sources of sediment has been successfully used throughout the world; however, such studies in Brazil are still incipient. This study aims to estimate the proportion of contribution of the major sources of sediment production from a hillside catchment area with the predominance of shallow soils and less intensive family agriculture. This catchment area is located in the central region of the State of Rio Grande do Sul, and the predominant land use is native forest, followed by native grassland, shrubland, annual crops, and a few areas of forestry (eucalyptus) and urbanized development. For identification of the sources of erosion, the fingerprinting method was used, which compares the soil from different sources and the sediments found in suspension in the drainage canal, using tracer elements. The largest contributions in sediment production are derived from soil surface erosion. For the first monitoring period, relative sediment production of $60 \%$ from unpaved roads at point T2, $100 \%$ of the sampling grid at point T3, and $77 \%$ of the sampling grid at point T4 was identified. For the second monitoring period, unpaved roads had a relative contribution of $81 \%$ of the suspended sediments at point T2 and $76 \%$ at point T3. For point T4, the greatest contribution was the sampling grid with $82 \%$. The method was sensitive enough to detect changes in the contribution of each source due to the characteristics they presented for the type of catchment area studied.
\end{abstract}

Index terms: fingerprinting, tracer elements, water erosion.

\section{INTRODUÇÃO}

A degradação dos recursos naturais pela ação antrópica vem se intensificando nas últimas décadas. As atividades agropecuárias estão entre as que mais perturbam o meio ambiente, pois expõem o solo à ação dos processos erosivos, acelerando a transferência de sedimentos aos corpos de água, juntamente com moléculas de agrotóxicos e fertilizantes, resultando na degradação do solo e da água (Gonçalves et al., 2005). O uso do solo fora da sua aptidão agrícola potencializa essas perturbações, tornando esses sistemas de produção frágeis e insustentáveis (Miguel et al., 2011). Além disso, a distribuição e a posição das lavouras e estradas na paisagem são tão ou mais importantes nas transferências de sedimentos do que a sua contribuição relativa em termos de área (Pellegrini et al., 2010).

Vários estudos que avaliam as perdas de solos e a geração de sedimentos utilizam parcelas padrões sob chuva simulada ou natural para estudar a dinâmica do processo erosivo e suas implicações ambientais. Esses trabalhos comparam diferentes tipos de sistemas de cobertura e preparo do solo, classes de declividade e tipos de solos (Cogo et al., 1983, 2003; Bertol et al.,
1997; 2007; 2010). Ao logo dos anos, esses trabalhos têm contribuído muito para a compreensão do processo erosivo e planejamento de práticas conservacionistas do solo.

Atualmente, novas formas de conduzir estudos de avaliação da geração de sedimentos vêm sendo utilizadas. Existem diferentes fontes de sedimentos que são transportadas em suspensão pelos canais fluviais (Minella et al., 2009a), sendo que, cada uma das fontes contribui de maneira variável em decorrência dos processos erosivos que ocorrem dentro da bacia hidrográfica. A identificação das fontes de sedimentos é de crucial importância para que se possa conceber uma gestão mais eficaz na redução das cargas de sedimentos (Walling, 2005).

O fato de que cálculos quantitativos para estimativa da proporção das fontes estão contribuindo para os sedimentos nos rios são mais úteis do que descrições puramente qualitativas foi elucidado com o trabalho de Yu \& Oldfield (1989). Os sedimentos em suspensão são originados de diferentes fontes e carregam com si características geoquímicas trazidas delas. Dessa forma, é possível traçar a origem dos sedimentos e a proporção com que cada fonte contribui por meio da avaliação de suas características 
geoquímicas. O estudo de traçadores geoquímicos é o mais difundido nos trabalhos de identificação de fontes de produção de sedimentos (Bird et al., 2010; Cui et al., 2010). Porém, esse método pode ser aplicado usando características traçantes naturais, como cor (Martínez-Carreras et al., 2010), suscetibilidade magnética (Yu \& Oldfield, 1989; Guzmán et al., 2010; Kim et al., 2013), mineralogia (Wall \& Wilding, 1976; He et al., 2012), ou utilizando elementos artificiais como os radionucleotídeos ${ }^{137} \mathrm{Cs},{ }^{7} \mathrm{Be}$ e ${ }^{210} \mathrm{~Pb}$ (Du et al., 2010).

Nos últimos anos, o método fingerprinting para identificação de fontes de sedimentos tem sido utilizado com sucesso no mundo todo (Walling et al., 2008; Ballantine et al., 2009; Martínez-Carreras et al., 2010; Bird et al., 2010; Collins et al., 2010a,b,c; Collins et al., 2012). Todavia, trabalhos dessa natureza realizados no Brasil ainda são incipientes. No Estado do Rio Grande do Sul, RS, foram desenvolvidos alguns trabalhos (Minella et al., 2007; 2009a,b), os quais verificaram que o método fingerprinting foi eficiente em detectar alterações na proporção de contribuição das fontes na produção sedimentos.

Os trabalhos existentes no Brasil priorizam bacias hidrográficas onde predominam solos profundos e que são intensamente cultivados (Minella et al., 2007, 2009a,b; Minella \& Merten, 2011). O objetivo deste trabalho foi estimar a proporção de contribuição das principais fontes de produção de sedimentos de uma bacia hidrográfica de encosta, onde predominam solos rasos e agricultura menos intensiva no sistema familiar.

\section{MATERIAL E MÉTODOS}

\section{Caracterização da área em estudo}

O estudo abrange uma área de aproximadamente $20 \mathrm{~km}^{2}$ da bacia hidrográfica do rio Vacacaí-Mirim, localizada na transição entre as regiões fisiográficas da Depressão Central e do Planalto Médio do Estado do Rio Grande do Sul, RS, chamada Rebordo do Planalto Sul $\left(53^{\circ} 35^{\prime} \mathrm{W}-54^{\circ} 00^{\prime} \mathrm{W}\right.$ e $\left.29^{\circ} 32^{\prime} \mathrm{S}-29^{\circ} 40^{\prime} \mathrm{S}\right)$. A bacia ocupa parte dos municípios de Santa Maria e Itaara, onde o regime pluvial anual médio é de 1.500 a $1.750 \mathrm{~mm}$, com a temperatura média anual de $19,4^{\circ} \mathrm{C}$. A água captada pela bacia por meio de sua drenagem principal, o rio Vacacaí-Mirim, abastece o reservatório da companhia Rio-Grandense de Saneamento (CORSAN), que é a responsável por parte do abastecimento de água da cidade de Santa Maria. Ao norte da área, em altitudes mais elevadas, estão localizadas as nascentes e ocorre o predomínio de rochas vulcânicas e solos mais desenvolvidos. Nas altitudes mais baixas, predominam rochas sedimentares e a topografia varia de suave ondulada a ondulada, com a ocorrência de solos de textura arenosa.
A bacia hidrográfica caracteriza-se por diferentes usos e tipos de solo. Mais de 50 \% da área têm ocupação do solo com floresta nativa seguida de áreas de campo, que englobam campo limpo, campo sujo e capoeira, ocupando $35 \%$ da área; lavouras anuais em $5 \%$ da área, em menor expressão silvicultura (eucalipto) e área urbanizada (Samuel-Rosa et al., 2011). Em mais de $50 \%$ da área, são encontrados solos pouco desenvolvidos, Neossolos Litólicos, geralmente em locais de maior declividade. Nas áreas localizadas mais ao norte da bacia de captação, são encontrados solos mais profundos, como Argissolos Vermelhos, numa proporção de $12 \%$; e, em locais com características de hifromorfismo, Planossolos Háplicos, pouco expressivos, com participação de 1,6 \% da área (Miguel et al., 2011).

\section{Coleta das amostras das fontes de sedimentos}

As fontes de produção de sedimentos analisadas na área foram estradas, canais fluviais e amostras de solo dos primeiros $20 \mathrm{~cm}$ do horizonte A (Figura 1a), abrangendo os diferentes usos do solo encontrados na área (Samuel-Rosa et al., 2011). Para cada local de coleta de sedimento em suspensão, o que corresponde a uma subdivisão da bacia (sub-bacia), foram coletadas no mínimo seis amostras de cada fonte. Para isso, foram consideradas a geologia da área de estudo (Figura 1b) (Maciel Filho, 1990), as classes de solos encontrados (Figura 1c) (Miguel et al., 2011) e os levantamentos de identificação de usos do solo (Figura $1 d$ ) e de processos erosivos predominantes (SamuelRosa et al., 2011; Miguel et al., 2011). As amostragens das fontes estradas e canais foram realizadas onde pôde ser observada a atuação de processos que caracterizavam a remoção de material. Nas estradas, as amostras foram mais representativas de horizontes subsuperficiais, bem como nos canais onde o rio imprime maior energia para retirada de material das margens.

A avaliação das perdas de sedimentos da área nos diferentes usos do solo que se encontram no local (Miguel et al., 2011) foi feita com base em uma malha de amostragem da parte superficial do solo $(20 \mathrm{~cm})$, com 340 pontos (Samuel-Rosa et al., 2011). Portanto, essa fonte, que é denominada neste trabalho como "malha", faz uma caracterização da situação de uso do solo na bacia hidrográfica de estudo e considera a perda de sedimento de toda a bacia, levando em consideração a parte superficial do solo.

\section{Coleta das amostras de sedimentos em suspensão}

Para a coleta de sedimentos em suspensão, foram instalados coletores em quatro pontos (T1, T2, T3 e T4) ou seções do rio Vacacaí-Mirim, que é a drenagem principal da bacia (Figura 1a). A avaliação, bem como a determinação da proporção de cada fonte, foi realizada para cada ponto de coleta separadamente. Salienta-se que cada ponto de coleta englobava uma 
divisão da bacia ou uma sub-bacia, assim, à medida que foi analisado cada um dos pontos, as amostras das fontes foram somadas em cada coletor. Dessa maneira, para a análise do coletor 4 (T4), foram utilizadas todas as amostras das fontes coletadas na bacia hidrográfica.

Os equipamentos de coleta de sedimentos consistem em um cano de PVC "cap" de $75 \mathrm{~mm} / 80 \mathrm{~cm}$, fechados em ambas as extremidades e com pequenos orifícios em cada ponta para a entrada de material, colocado dentro da calha do rio. O período de monitoramento de coleta desse sedimento foi de três a quatro meses, tempo estimado para que se obtenha quantidade suficiente de material para a análise, sendo a $1^{\mathrm{a}}$ coleta com instalação no dia 15 de maio de 2011 e coleta do sedimento no dia 15 de agosto de 2011; e a $2^{\text {a }}$ coleta no dia 15 de dezembro de 2011.

\section{Análises físicas}

Após coletadas, as amostras foram secas ao ar; posteriormente, determinou-se a granulometria. Neste trabalho, foi considerado um padrão de granulometria para os procedimentos de análises químicas e mineralógicas de $150 \mu \mathrm{m}$, pelo fato de mais de $90 \%$ da granulometria do sedimento estar abaixo desse diâmetro de partícula. Sendo assim, para o fator de correção, foi determinada a composição granulométrica de todas as amostras das fontes e do sedimento em

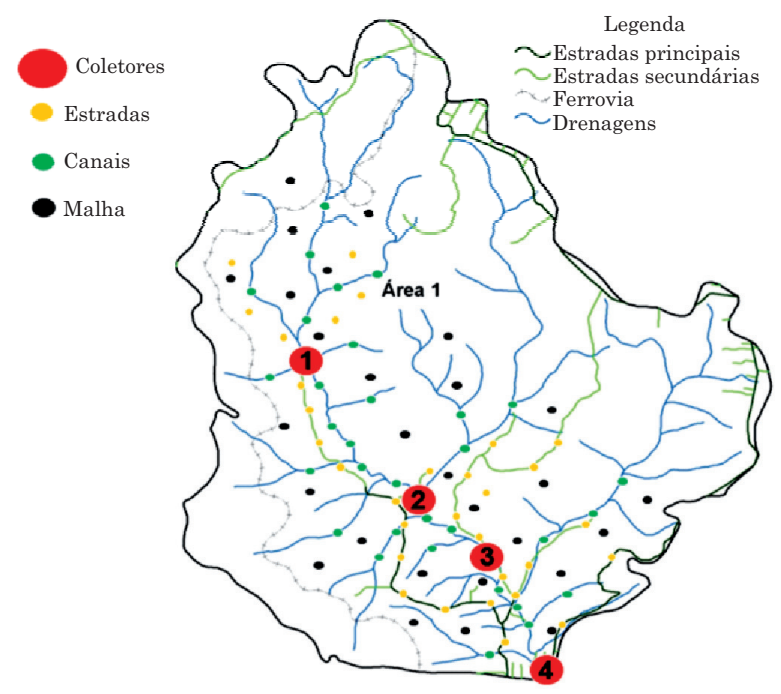

(a)

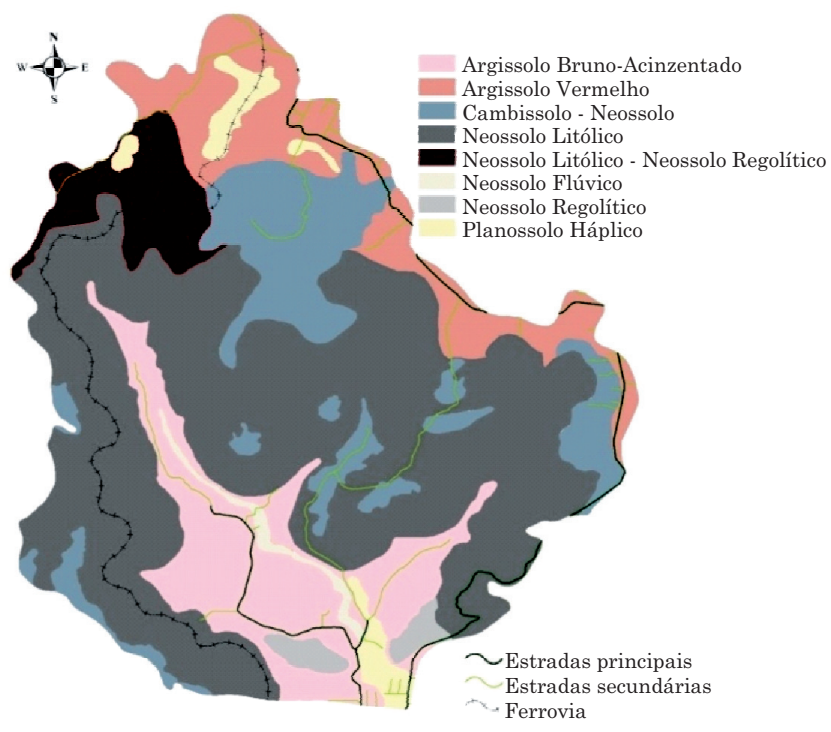

(c)
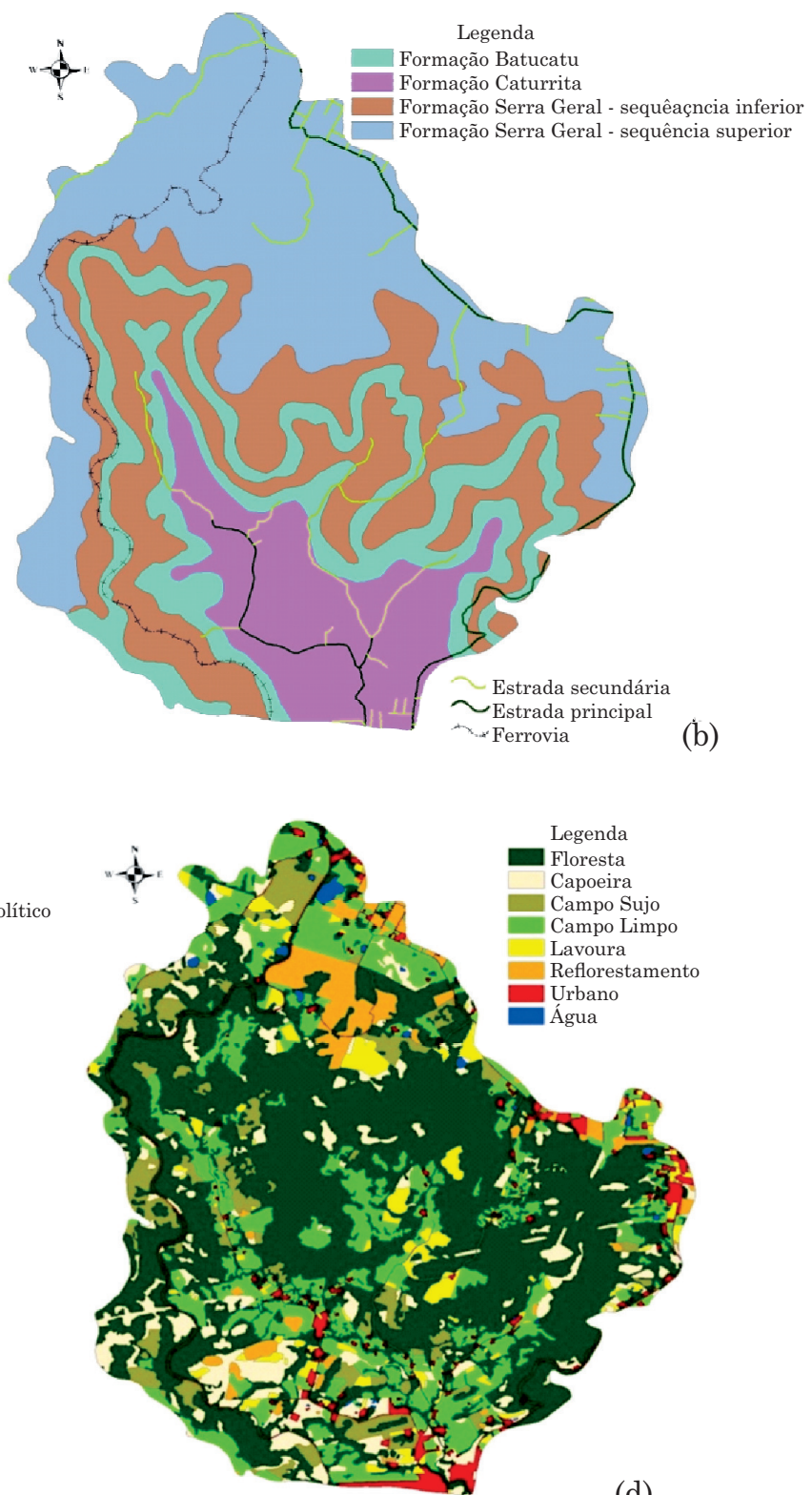

(d)

Figura 1. a) Área de estudo com indicação dos locais das coletas das fontes de produção de sedimentos e dos locais de instalação dos coletores de sedimentos em suspensão (CSS). b) Mapa geológico (Maciel Filho, 1990), c) Mapa pedológico (Miguel et al., 2011), d) Mapa de uso da terra de 2008/2009 (Samuel Rosa et al., 2011). 
suspensão, utilizando granulômetro a laser marca Beckman Coulter LS 13 320, após dispersão química das amostras e $12 \mathrm{~h}$ de agitação. Como a concentração dos elementos químicos é dependente da granulometria dos sedimentos em suspensão, Horowitz (1991) propôs uma correção das concentrações utilizando-se a fórmula: $Z_{s i}=C_{o}\left[100 /\left(100-F_{\text {ars }}\right)\right]$, em que $Z_{s i}$ é a concentração corrigida das fontes e do elemento químico analisado $(i) ; C_{o}$, a concentração media original do elemento químico analisado; e $F_{\text {ars }}$, a porcentagem da fração maior que $63 \mu \mathrm{m}$ das amostras da fonte. Para o cálculo da fórmula de Horowitz (1991), foi utilizada a porcentagem de areia fina $\left(\mathrm{F}_{\mathrm{ars}}\right)$, ou seja, o valor encontrado entre a fração 63 e $150 \mu \mathrm{m}$. Dessa forma, corrigindo a concentração dos elementos químicos nos sedimentos e no solo das fontes, a comparação entre esses pode ser possível e a incerteza relacionada ao enriquecimento dos sedimentos com partículas finas pode ser contornada ou minimizada.

\section{Análises químicas}

As amostras das fontes e o sedimento em suspensão depois de passar por peneira com abertura de $150 \mu \mathrm{m}$ foram submetidas à digestão com ácido nítrico $\left(\mathrm{HNO}_{3}\right)$ + ácido clorídrico $(\mathrm{HCl})$, segundo o método $\mathrm{n}^{\circ} 3050 \mathrm{~B}$ (EPA, 1996). Os teores de Al, Sb, Ba, Be, Cd, Ca, Cl, $\mathrm{Co}, \mathrm{Cu}, \mathrm{P}, \mathrm{Fe}, \mathrm{Pb}, \mathrm{Mg}, \mathrm{Mn}, \mathrm{Mo}, \mathrm{Ni}, \mathrm{K}, \mathrm{Ag}, \mathrm{Na}, \mathrm{Zn}$, TI, e V nesses extratos foram determinados por Espectrofotometria de Plasma Indutivamente Acoplado (ICP).

Para determinar os teores e a composição química dos óxidos de baixa e alta cristalinidade, foram utilizados os métodos do oxalato de amônio $0,2 \mathrm{~mol} \mathrm{~L}^{-1}$, $\mathrm{pH}$ 3,0 (OAA) e do ditionito-citrato-bicarbonato (DCB). Após as extrações, os teores de $\mathrm{Fe}, \mathrm{Mn}$ e $\mathrm{Al}$ foram determinados por espectrofotometria de absorção atômica. $\mathrm{O}$ teor de carbono orgânico total (COT) foi obtido pelo método Mébius no bloco de digestão (Yeomans \& Bremner, 1988). Considerando as análises químicas, 29 variáveis foram analisadas para caracterizar o solo das fontes e do sedimento e usadas também para os procedimentos estatísticos.

\section{Discriminação das fontes de sedimentos}

Após as determinações das características físicas e químicas, realizaram-se os seguintes procedimentos estatísticos: a primeira etapa da análise estatística constituiu em estabelecer o conjunto de variáveis com capacidade de discriminar as fontes, ou seja, as variáveis precisam apresentar diferenças significativas na sua concentração entre as fontes a serem identificadas.

Para isso, foram utilizados dois testes estatísticos: o teste não paramétrico Kruskal-Wallis $(H)$ e uma função discriminante multivariada (Collins et al., 1997; Collins \& Walling, 2002). O teste H permitiu testar a hipótese nula de que as fontes são provenientes de uma mesma população. Nesse teste, foram definidos que elementos químicos apresentaram diferença estatística entre as fontes de sedimentos, os quais puderam ser utilizados como variáveis traçadoras. O teste foi aplicado para cada variável geoquímica, verificando sua capacidade individual em discriminar as fontes (Equação 1).

$$
\mathrm{H}=\frac{12}{\mathrm{n}(\mathrm{n}+1)} \sum_{\mathrm{S}=1}^{\mathrm{K}} \frac{\mathrm{R}_{\mathrm{i}}^{2}}{\mathrm{n}_{\mathrm{i}}}-3(\mathrm{n}+1)
$$

em que $R i$ se refere à soma dos "ranks" ocupados pela fonte s, sendo $n_{i}$ o número de observações da fonte $s$; $n$, à soma $\operatorname{dos} n_{i}$ 's; e $k$, ao número de fontes.

$\mathrm{O}$ segundo teste foi uma função discriminante multivariada que determina o número mínimo de variáveis que maximiza a discriminação das fontes. O método é com base no parâmetro Lambda de Wilks $\left(\Lambda^{*}\right)$ da análise de variância multivariada, e o critério utilizado pelo modelo estatístico é a minimização de $\Lambda^{*}$ (Johnson \& Wichern, 1992) (Equação 2). Não existe capacidade discriminante (as fontes são consideradas iguais) do conjunto de variáveis selecionadas quando $\Lambda^{*}$ é próximo de 1 . Valores próximos de 0 ocorrem quando a variabilidade dentro do grupo é pequena, em comparação com a variabilidade total; isto é, a maior parte da variabilidade total pode ser atribuída à diferença entre os grupos.

$$
\Lambda=\frac{|\mathrm{W}|}{|\mathrm{B}+\mathrm{W}|}
$$

em que $|\mathrm{W}|$ é o determinante da matriz da soma dos quadrados em razão do erro, enquanto $|\mathrm{B}+\mathrm{W}|$ representa o determinante da matriz da soma dos quadrados totais.

Na equação 3, descreve-se a relação matemática entre as proporções das fontes e as variáveis das fontes e dos sedimentos em suspensão (Walling \& Woodward, 1995).

$$
\mathrm{y}_{\mathrm{i}}=\sum_{\mathrm{s}=1}^{\mathrm{n}} \mathrm{a}_{\mathrm{is}} \mathrm{P}_{\mathrm{s}}(\mathrm{s}=1,2, \ldots, \mathrm{n}) \mathrm{e}(\mathrm{i}=1,2, \ldots, \mathrm{m})
$$

em que $y_{i}$ é o valor da variável i obtida no sedimento em suspensão, $a_{i s}$ são os coeficientes do modelo linear (concentração do elemento químico i na fonte s) e $P_{s}$ é a proporção da massa da fonte $s$, que podem ser apresentados como um conjunto de funções lineares de $m$ variáveis e $n$ fontes.

Para determinar os valores de $P$, foi utilizada uma função objetivo (Walling \& Woodward, 1995). A solução é encontrada por meio de um processo interativo, objetivando minimizar o valor de $R$ ( $f$ mincon) (Equação 4). No processo de minimização, os valores de $P$ ficam sujeitos a duas restrições: serem maiores ou iguais a zero e menores ou iguais a 1 (Equação 5); e a soma $\operatorname{dos} P_{s}$ deve ser igual a 1 (Equação 6).

$$
\mathrm{R}=\sum_{\mathrm{i}=1}^{\mathrm{m}}\left\{\frac{\mathrm{C}_{\mathrm{i}}-\left(\sum_{\mathrm{s}=1}^{\mathrm{n}} \mathrm{P}_{\mathrm{s}} \mathrm{C}_{\mathrm{si}} \mathrm{Z}_{\mathrm{s}}\right)}{\mathrm{C}_{\mathrm{i}}}\right\}^{2}
$$




$$
\begin{gathered}
0 \leq \mathrm{P}_{\mathrm{s}} \leq 1^{\prime} \\
\sum_{\mathrm{s}=1}^{\mathrm{g}} \mathrm{P}_{\mathrm{s}}=1
\end{gathered}
$$

em que $m$ é o número de elementos químicos selecionados como variáveis traçadoras; $n$, o número de fontes; $C i$, a concentração da variável traçadora $i$ na amostra de sedimento em suspensão; $P_{s}$, a proporção de contribuição da fonte $s ; C_{s i}$, o valor médio da variável traçadora $i$ obtido na fonte $s$; e $Z_{s}$, o fator de correção granulométrica da amostra da fonte $s$.

Para confirmar que o processo de otimização da equação 4 forneceu resultados aceitáveis, foram comparados os resultados da concentração química dos elementos utilizados (variáveis traçadoras) nos sedimentos em suspensão e no valor predito pelo modelo, com base na proporção calculada para cada fonte. Com os valores do erro relativo de cada variável, foi calculado um erro médio relativo (ERM) para providenciar um valor único associado a cada amostra de sedimentos em suspensão (Equação 7).

$$
\mathrm{ERM}=\sum_{\mathrm{i}=1}^{\mathrm{m}}\left\{\frac{\mathrm{C}_{\mathrm{i}}-\left(\sum_{\mathrm{s}=1}^{\mathrm{n}} \mathrm{P}_{\mathrm{s}} \mathrm{C}_{\mathrm{si}}\right)}{\mathrm{m}}\right\}
$$

De acordo com Walling \& Collins (2000), quando o resultado da equação 7 apresentar valores menores que $15 \%$, evidencia-se que o modelo encontrou uma solução viável dos valores de $P_{s}$ (contribuições relativas de cada fonte), provenientes do procedimento de minimização da equação 4 .

\section{RESULTADOS E DISCUSSÃO}

\section{Potencial discriminante das fontes}

Das 29 variáveis analisadas nas amostras das fontes, pela aplicação do teste Kruskal-Wallis, em nível de significância de $90 \%\left(H_{\text {crítico }}=4,60\right)$, somente algumas foram selecionadas. O teste de Kruskal-Wallis selecionou diferentes variáveis em cada local de coleta (Quadros 1, 2, 3 e 4). Para o T1, as variáveis selecionadas foram $\mathrm{Sr}$, Ti, COT, $\mathrm{Fe}_{\text {oxa }}, \mathrm{Al}_{\text {oxa }}$ e $\mathrm{Al}_{\mathrm{dcb}}$. Variáveis relacionadas aos óxidos, cristalinos ou amorfos, evidenciam fatores de intemperismo e reações de oxidação-redução. O COT, apesar de ser uma variável que pode apresentar problemas na análise pela sua instabilidade no sistema e pelo grande enriquecimento que pode ser encontrado no sedimento em suspensão (Minella et al., 2009a), foi utilizado como variável-traço, por se encontrar dentro dos limites em que os fatores de correção puderam corrigir, estando associado a fatores de manejo do solo. Para o T2, as variáveis que apresentaram capacidade discriminante foram $\mathrm{Ba}, \mathrm{Mg}$, La, Sr, Ti, Pb, Na, Be, P e COT. Para o T3, foram Ca, Ba, Mg, La, Sr, Zn, Ti, Pb, Na, Be, $\mathrm{P}, \mathrm{V}, \mathrm{COT}, \mathrm{Fe}, \mathrm{Fe}_{\text {oxa }}$, Mn, $\mathrm{Mn}_{\text {oxa }}$ e $\mathrm{Mn}_{\mathrm{dcb}}$. Para o T4, $75 \%$ das variáveis apresentaram capacidade discriminante, ficando fora da análise somente as variáveis como $\mathrm{K}, \mathrm{Cr}, \mathrm{Co}, \mathrm{Si}, \mathrm{Mn}_{\text {oxa }}, \mathrm{Al}_{\text {oxa }}$ e $\mathrm{Al}_{\mathrm{dcb}}$.

Algumas variáveis demonstraram capacidade discriminante em todos os pontos de coleta avaliados. O COT, Ti e Sr aparecem como variáveis discriminantes em todas as análises. Elementos como $\mathrm{Ba}, \mathrm{Mg}$, La, Sr, Ti, Pb, Na, Be e P evidenciaram capacidade discriminante para os pontos de coleta T2, T3, e T4. Deve-se ter cuidado com elementos de maior instabilidade como o $\mathrm{Na}$, por exemplo, pois esse pode sofrer modificações como enriquecimentos de seus teores nos sedimentos em suspensão, comprometendo assim a análise de contribuição de cada fonte.

$\mathrm{O} P$ é um elemento que não apresenta capacidade discriminante para o ponto $\mathrm{T} 1$. Como esse elemento está associado a condições de ocupação agrícola nas bacias e em áreas de lavoura, por exemplo, onde são aplicados adubos fosfatados, facilmente ocorre a diferenciação entre as fontes (Minella et al., 2007). Mesmo que o ponto T1 possua uma coleta de sedimento em suspensão de uma sub-bacia com ocupação agrícola maior, comparada aos demais locais, visto a maior concentração média do elemento nas fontes para o ponto T1, a quantidade do elemento não diferiu entre as fontes. Contudo, para os demais pontos de coletas (T2, T3 e T4), o P se apresentou como uma variável com poder de discriminação, visto que esse elemento apresenta relação com partículas de argila e óxidos, o que o torna bom traçador de sedimentos em suspensão (Minella et al., 2007). Os elementos Zn, Ti, V, Fe, $\mathrm{Fe}_{\text {oxa }}, \mathrm{Fe}_{\mathrm{dcb}}, \mathrm{Mn}, \mathrm{Mn}_{\text {oxa }}$ e $\mathrm{Mn}_{\mathrm{dcb}}$, classificados como metais de transição, evidenciaram capacidade discriminante entre as fontes e geralmente apresentam boa conservatividade (Horowitz, 1991), ou seja, tem a capacidade de manter as suas características mesmo quando removidos e transportados até a calha dos rios. Entre os metais de transição, o $\mathrm{Cr}$, Co, $\mathrm{Ni}$ e $\mathrm{Cu}$ não apresentaram capacidade discriminante.

Foi realizada a determinação do parâmetro Wilk's Lambda $\left(\Lambda^{*}\right)$ para a avaliação dos resultados da análise de variância multivariada e determinação do grupo de variáveis que otimiza a discriminação das fontes (Quadro 5). As variáveis foram testadas na análise de variância e aquelas que apresentaram menores valores de $\Lambda^{*}$ foram selecionadas para 0 modelo de classificação. Pela análise discriminante, pode ser observado aumento no conjunto de variáveis de minimização do parâmetro $\Lambda^{*}$. Isso se deve ao aumento da quantidade de amostras analisadas em cada uma das análises. Por exemplo, para o local T1, foram analisadas 20 amostras divididas em três fontes e, para o local T4, 90 amostras distribuídas dentro das mesmas três fontes de produção de sedimentos.

$O$ valor de $\Lambda^{*}$ é definido como a proporção da variabilidade total das fontes por causa do erro. Dessa 
maneira, para o local de coleta $\mathrm{T} 1$, o conjunto de variáveis selecionadas reduziu o erro para $3,8 \%$, ou seja, esse conjunto de variáveis explica, aproximadamente, $96 \%$ das diferenças entre as fontes. Para os demais locais, ocorreu aumento no erro com maior número de variáveis selecionadas, erro esse de aproximadamente $14 \%$. É importante que exista grande número de variáveis que apresentem diferença entre as fontes, pois cada uma delas pode estar relacionada a um tipo de erosão (Small et al., 2002). Contudo, segundo Minella et al. (2007), isso pode dificultar a análise na obtenção da contribuição de cada fonte, por isso a importância de otimizar o conjunto de elementos a ser utilizado e maximizar a discriminação com o menor número possível de variáveis.

Para correção dos efeitos de diluição do material grosseiro das fontes, foi realizado o cálculo do fator de correção (Quadro 6), sendo possível assim a comparação dos teores dos elementos químicos das fontes com os dos sedimentos em suspensão. Essa necessidade de correção está atrelada ao fato de que a granulometria dos sedimentos pode apresentar distintas capacidades de adsorção de produtos químicos (Bertol et al., 2007), influenciando, assim, a seleção de sistemas de manejo e de práticas conservacionistas do solo. A erosão hídrica transporta preferencialmente os sedimentos mais finos e de baixa densidade, constituídos, sobretudo, de

Quadro 1. Resultados do teste de Kruskal-Wallis para cada variável e teor de cada variável no solo das fontes no local de coleta T1 (Coletor 1)

\begin{tabular}{|c|c|c|c|c|c|}
\hline \multirow{2}{*}{ Variável } & \multicolumn{2}{|c|}{ Teste Kruskal-Wallis } & \multicolumn{3}{|c|}{ Concentração média nas fontes } \\
\hline & $H^{(1)}$ & $\mathbf{p}^{(2)}$ & Estrada & Canal & Malha \\
\hline \multicolumn{6}{|l|}{ Teor } \\
\hline $\mathrm{Al}$ & 3,073 & 0,2151 & 39,762 & 25,664 & 29,060 \\
\hline $\mathrm{Fe}$ & 0,551 & 0,7592 & 43,913 & 33,830 & 38,341 \\
\hline $\mathrm{Ca}$ & 1,716 & 0,4239 & 2,208 & 3,227 & 2,598 \\
\hline $\mathrm{Ba}$ & 1,998 & 0,3681 & 0,150 & 0,203 & 0,225 \\
\hline $\mathrm{Mg}$ & 1,770 & 0,4127 & 4,331 & 4,877 & 4,102 \\
\hline $\mathrm{La}$ & 0,461 & 0,7940 & 0,021 & 0,022 & 0,019 \\
\hline $\mathrm{Li}$ & 0,681 & 0,7112 & 0,023 & 0,019 & 0,020 \\
\hline Mn & 4,083 & 0,1298 & 1,217 & 1,129 & 2,130 \\
\hline $\mathrm{Ni}$ & 3,457 & 0,1775 & 0,011 & 0,009 & 0,012 \\
\hline $\mathrm{Sr}$ & 12,110 & $0,0023^{*}$ & 0,027 & 0,094 & 0,047 \\
\hline $\mathrm{Zn}$ & 1,538 & 0,4633 & 0,027 & 0,036 & 0,035 \\
\hline K & 0,823 & 0,6626 & 1,883 & 1,863 & 2,021 \\
\hline $\mathrm{Ti}$ & 5,678 & $0,0585^{*}$ & 3,973 & 5,158 & 4,712 \\
\hline $\mathrm{Pb}$ & 2,940 & 0,2298 & 0,008 & 0,014 & 0,009 \\
\hline $\mathrm{Cu}$ & 3,729 & 0,1550 & 0,039 & 0,056 & 0,055 \\
\hline $\mathrm{Cr}$ & 3,182 & 0,2037 & 0,014 & 0,011 & 0,014 \\
\hline $\mathrm{Na}$ & 0,589 & 0,7446 & 0,203 & 0,211 & 0,192 \\
\hline $\mathrm{Be}$ & 0,078 & 0,9616 & 0,002 & 0,001 & 0,002 \\
\hline Co & 1,324 & 0,5157 & 0,029 & 0,024 & 0,039 \\
\hline $\mathrm{P}$ & 3,474 & 0,1760 & 0,323 & 0,326 & 0,465 \\
\hline $\mathrm{Si}$ & 3,073 & 0,2151 & 0,164 & 0,134 & 0,135 \\
\hline V & 1,157 & 0,5605 & 0,150 & 0,180 & 0,174 \\
\hline $\mathrm{COT}^{(3)}$ & 12,256 & $0,0022^{*}$ & 9,825 & 25,022 & 26,980 \\
\hline \multicolumn{6}{|c|}{ Dissolução seletiva $^{(4)}$} \\
\hline $\mathrm{Fe}(\mathrm{DCB})$ & 0,029 & 0,9855 & 30,864 & 32,000 & 27,164 \\
\hline Fe (oxa) & 13,221 & $0,0013^{*}$ & 2,664 & 3,704 & 11,322 \\
\hline $\mathrm{Mn}(\mathrm{DCB})$ & 4,088 & 0,1295 & 0,934 & 1,088 & 0,801 \\
\hline Mn (oxa) & 4,280 & 0,1176 & 0,476 & 0,134 & 0,079 \\
\hline $\mathrm{Al}(\mathrm{DCB})$ & 5,100 & $0,0781^{*}$ & 3,105 & 2,630 & 2,034 \\
\hline $\mathrm{Al}$ (oxa) & 8,818 & $0,0122^{*}$ & 2,116 & 2,362 & 1,531 \\
\hline
\end{tabular}

(1) Valor do teste de discriminação que se aproxima dos valores da distribuição qui-quadrado - $\mathrm{H}_{\text {crítico }}(90 \%$; e 2 gl) $=4,60$; (2) probabilidade de que as fontes diferem entre si, segundo a distribuição qui-quadrado; * p<0,10; ${ }^{(3)}$ COT: carbono orgânico total e ${ }^{(4)}$ teores de elementos extraídos com Ditionito-citrato-bicarbonato - DCB (Mehra \& Jackson, 1960) e com Oxalato de amônio - oxa (Schwertmann, 1964). 
coloides minerais e orgânicos e enriquecidos de elementos minerais, apresentando alta atividade química (Bertol et al., 2007). Os fatores que mais explicam a distribuição de tamanho de sedimentos transportados pela enxurrada (Cogo et al., 1983; Bertol et al., 1997, 2010) são a velocidade com que essa ocorre e a cobertura do solo.

Neste estudo, foi considerado como material não reativo a fração maior que $150 \mu \mathrm{m}$. Para o fator de correção, foi utilizada a porcentagem de diferença entre as frações 150 e $63 \mu \mathrm{m}$, que, segundo Horowitz (1991), seriam as partículas primárias, corrigindo assim as concentrações químicas das fontes por meio dessa proporção.

O fato para que fosse usado tal limite de granulometria está relacionado com a característica do sedimento coletado. A maior proporção do sedimento (90 \%) ficou concentrada em diâmetro menor que 150 $\mu \mathrm{m}$. Isso se deve a características dos tipos de solos que são encontrados na bacia, visto que, segundo Miguel et al. (2011), os solos que predominam são os de textura mais arenosa, principalmente no horizonte superficial, como os Argissolos. Até mesmo os solos menos desenvolvidos da área apresentam essa

Quadro 2. Resultados do teste de Kruskal-Wallis para cada variável e teor de cada variável no solo das fontes no local de coleta T2 (Coletor 2)

\begin{tabular}{|c|c|c|c|c|c|}
\hline \multirow{2}{*}{ Variável } & \multicolumn{2}{|c|}{ Teste Kruskal-Wallis } & \multicolumn{3}{|c|}{ Concentração média nas fontes } \\
\hline & $H^{(1)}$ & $\mathbf{p}^{(2)}$ & Estrada & Canal & Malha \\
\hline & & & & $-\mathrm{g} \mathrm{kg}^{-1}$ & \\
\hline \multicolumn{6}{|l|}{ Teor } \\
\hline $\mathrm{Al}$ & 3,409 & 0,1818 & 34,065 & 30,992 & 24,720 \\
\hline $\mathrm{Fe}$ & 3,170 & 0,2049 & 36,745 & 35,562 & 27,563 \\
\hline $\mathrm{Ca}$ & 4,389 & 0,1114 & 2,577 & 2,855 & 2,610 \\
\hline $\mathrm{Ba}$ & 6,739 & $0,0344^{*}$ & 0,124 & 0,162 & 0,155 \\
\hline $\mathrm{Mg}$ & 7,947 & $0,0188^{*}$ & 4,484 & 4,732 & 4,209 \\
\hline $\mathrm{La}$ & 7,623 & $0,0221^{*}$ & 0,019 & 0,024 & 0,016 \\
\hline $\mathrm{Li}$ & 2,549 & 0,2795 & 0,021 & 0,021 & 0,016 \\
\hline $\mathrm{Mn}$ & 4,065 & 0,2160 & 0,948 & 1,084 & 1,484 \\
\hline $\mathrm{Ni}$ & 1,991 & 0,3694 & 0,020 & 0,013 & 0,013 \\
\hline $\mathrm{Sr}$ & 20,806 & $0,0000^{*}$ & 0,042 & 0,063 & 0,037 \\
\hline $\mathrm{Zn}$ & 3,946 & 0,1390 & 0,023 & 0,028 & 0,025 \\
\hline $\mathrm{K}$ & 0,938 & 0,6255 & 1,744 & 1,934 & 2,018 \\
\hline $\mathrm{Ti}$ & 10,018 & $0,0067^{*}$ & 3,770 & 4,587 & 4,003 \\
\hline $\mathrm{Pb}$ & 14,736 & $0,0006^{*}$ & 0,006 & 0,010 & 0,008 \\
\hline $\mathrm{Cu}$ & 1,143 & 0,5646 & 0,065 & 0,054 & 0,050 \\
\hline $\mathrm{Cr}$ & 1,073 & 0,5847 & 0,017 & 0,014 & 0,013 \\
\hline $\mathrm{Na}$ & 6,133 & $0,0466^{*}$ & 0,342 & 0,239 & 0,230 \\
\hline $\mathrm{Be}$ & 5,314 & $0,0702^{*}$ & 0,002 & 0,002 & 0,001 \\
\hline Co & 0,112 & 0,9453 & 0,027 & 0,028 & 0,030 \\
\hline $\mathrm{P}$ & 5,670 & $0,0587^{*}$ & 0,237 & 0,290 & 0,351 \\
\hline $\mathrm{Si}$ & 0,439 & 0,8028 & 0,139 & 0,151 & 0,134 \\
\hline $\mathrm{V}$ & 4,455 & 0,1078 & 0,168 & 0,186 & 0,136 \\
\hline $\mathrm{COT}^{(3)}$ & 24,914 & $0,0000^{*}$ & 10,053 & 15,376 & 22,787 \\
\hline \multicolumn{6}{|c|}{ Dissolução seletiva ${ }^{(4)}$} \\
\hline $\mathrm{Fe}(\mathrm{DCB})$ & 1,610 & 0,4469 & 22,871 & 22,356 & 20,807 \\
\hline $\mathrm{Fe}$ (oxa) & 2,058 & 0,3572 & 1,377 & 4,812 & 1,986 \\
\hline $\mathrm{Mn}(\mathrm{DCB})$ & 3,447 & 0,1784 & 0,717 & 0,901 & 0,963 \\
\hline Mn (оха) & 0,327 & 0,8491 & 0,439 & 0,439 & 0,379 \\
\hline $\mathrm{Al}(\mathrm{DCB})$ & 2,589 & 0,2740 & 3,264 & 2,170 & 2,249 \\
\hline $\mathrm{Al}$ (oxa) & 2,664 & 0,2639 & 1,788 & 1,303 & 1,720 \\
\hline
\end{tabular}

(1) Valor do teste de discriminação que se aproxima dos valores da distribuição qui-quadrado - $\mathrm{H}_{\text {crítico }}(90 \%$; e 2 gl) $=4,60$;

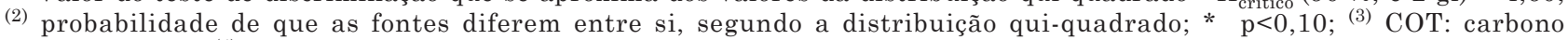
orgânico total e ${ }^{(4)}$ teores de elementos extraídos com Ditionito-citrato-bicarbonato - DCB (Mehra \& Jackson, 1960) e com Oxalato de amônio - oxa (Schwertmann, 1964). 
característica. O material de origem possui influência direta em razão de a área apresentar solos derivados de arenito da Formação Botucatu e da Formação Caturrita, conferindo aos solos tal característica textural.

\section{Classificação das amostras de sedimento em suspensão}

O modelo de classificação das amostras de sedimentos em suspensão somente não foi eficiente na minimização da função objetivo para o ponto T1, em ambas as coletas de sedimento. Para os demais, T2, T3 e T4, o modelo evidenciou-se satisfatório visto que os erros médios relativos foram menores que $15 \%$ (Figura 2). Para a primeira coleta de sedimentos, o modelo determinou uma contribuição na produção global de sedimentos de $100 \%$ da malha para o ponto T1. Nessa situação, não se pôde fazer uma análise do resultado, pois o erro ( $27 \%)$ foi maior que $15 \%$. Uma possível explicação pode ser que o número de amostras coletadas não foi elevado. $\mathrm{O}$ ponto $\mathrm{T} 2$ apresentou contribuição maior das estradas (81 \%) na segunda coleta em relação à primeira $(66 \%)$ e não demonstrou contribuição do canal. Esse ponto se concentra em uma

Quadro 3. Resultados do teste de Kruskal-Wallis para cada variável e teor de cada variável no solo das fontes no local de coleta T3 (Coletor 3)

\begin{tabular}{|c|c|c|c|c|c|}
\hline \multirow{2}{*}{ Variável } & \multicolumn{2}{|c|}{ Teste Kruskal-Wallis } & \multicolumn{3}{|c|}{ Concentração média nas fontes } \\
\hline & $H^{(1)}$ & $\mathbf{p}^{(2)}$ & Estrada & Canal & Malha \\
\hline & & & & $-\mathrm{g} \mathrm{kg}^{-1}$ & + \\
\hline \multicolumn{6}{|l|}{ Teor } \\
\hline $\mathrm{Al}$ & 2,506 & 0,2856 & 27,910 & 23,961 & 22,526 \\
\hline $\mathrm{Fe}$ & 4,661 & $0,0972^{*}$ & 26,816 & 28,485 & 24,055 \\
\hline $\mathrm{Ca}$ & 6,233 & $0,0443^{*}$ & 2,560 & 2,874 & 2,268 \\
\hline $\mathrm{Ba}$ & 11,102 & $0,0039^{*}$ & 0,106 & 0,167 & 0,145 \\
\hline $\mathrm{Mg}$ & 6,325 & $0,0423^{*}$ & 3,913 & 4,281 & 3,735 \\
\hline $\mathrm{La}$ & 7,227 & $0,0270^{*}$ & 0,021 & 0,024 & 0,018 \\
\hline $\mathrm{Li}$ & 4,417 & 0,1098 & 0,015 & 0,017 & 0,014 \\
\hline $\mathrm{Mn}$ & 11,639 & $0,0030^{*}$ & 0,657 & 0,974 & 1,259 \\
\hline $\mathrm{Ni}$ & 0,573 & 0,7505 & 0,015 & 0,010 & 0,011 \\
\hline $\mathrm{Sr}$ & 22,228 & $0,0000 *$ & 0,034 & 0,073 & 0,037 \\
\hline $\mathrm{Zn}$ & 9,361 & $0,0093^{*}$ & 0,019 & 0,027 & 0,023 \\
\hline $\mathrm{K}$ & 0,183 & 0,9123 & 1,871 & 1,742 & 2,091 \\
\hline $\mathrm{Ti}$ & 13,165 & $0,0014^{*}$ & 3,250 & 4,588 & 3,747 \\
\hline $\mathrm{Pb}$ & 15,959 & $0,0003^{*}$ & 0,006 & 0,011 & 0,008 \\
\hline $\mathrm{Cu}$ & 2,832 & 0,2426 & 0,050 & 0,051 & 0,043 \\
\hline $\mathrm{Cr}$ & 0,046 & 0,9771 & 0,014 & 0,012 & 0,012 \\
\hline $\mathrm{Na}$ & 7,175 & $0,0277^{*}$ & 0,311 & 0,210 & 0,188 \\
\hline $\mathrm{Be}$ & 8,480 & $0,0144^{*}$ & 0,001 & 0,002 & 0,001 \\
\hline $\mathrm{Co}$ & 3,917 & 0,1410 & 0,019 & 0,022 & 0,025 \\
\hline $\mathrm{P}$ & 12,785 & $0,0017^{*}$ & 0,189 & 0,254 & 0,321 \\
\hline $\mathrm{Si}$ & 0,208 & 0,9010 & 0,135 & 0,134 & 0,131 \\
\hline V & 8,688 & $0,0130^{*}$ & 0,128 & 0,170 & 0,119 \\
\hline $\mathrm{COT}^{(3)}$ & 33,299 & $0,0000^{*}$ & 8,997 & 18,054 & 20,863 \\
\hline \multicolumn{6}{|c|}{ Dissolução seletiva ${ }^{(4)}$} \\
\hline $\mathrm{Fe}(\mathrm{DCB})$ & 4,060 & 0,1313 & 17,650 & 20,807 & 17,714 \\
\hline $\mathrm{Fe}$ (оха) & 6,172 & $0,0457^{*}$ & 1,597 & 4,617 & 1,741 \\
\hline $\mathrm{Mn}$ (DCB) & 14,683 & $0,0006^{*}$ & 0,462 & 0,776 & 0,886 \\
\hline Mn (oxa) & 7,161 & $0,0279^{*}$ & 0,273 & 0,472 & 0,447 \\
\hline $\mathrm{Al}$ (DCB) & 0,144 & 0,9303 & 2,801 & 2,337 & 2,321 \\
\hline $\mathrm{Al}$ (oxa) & 3,876 & 0,1439 & 1,589 & 1,277 & 1,638 \\
\hline
\end{tabular}

(1) Valor do teste de discriminação que se aproxima dos valores da distribuição qui-quadrado - $\mathrm{H}_{\text {crítico }}(90 \%$; e 2 gl) $=4,60$; (2) probabilidade de que as fontes diferem entre si, segundo a distribuição qui-quadrado; * ${ }^{\text {p }<0,10 ; ~}{ }^{(3)}$ COT: carbono orgânico total e ${ }^{(4)}$ teores de elementos extraídos com Ditionito-citrato-bicarbonato - DCB (Mehra \& Jackson, 1960) e com Oxalato de amônio - oxa (Schwertmann, 1964). 
posição na bacia em que o rio Vacacaí-Mirim encontrase mais conservado, onde as margens se apresentam em melhores condições e são observados pontos isolados de assoreamento. As áreas de preservação permanente (APPs) têm papel importante nessa parte da bacia, se mantendo ainda na sua maior parte dentro da legislação, apesar de as propriedades existentes como sítios (Samuel-Rosa et al., 2011) possuírem suas construções próximas alguns metros da margem do rio. Segundo Miguel et al. (2011), os conflitos de uso do solos se concentram mais próximos ao reservatório da CORSAN, onde o uso antrópico é mais denso (Miguel et al., 2011), com problemas de ocupação, no entanto, o rio Vacacaí-Mirim não apresenta tantas mudanças no seu leito pela conservação das APPs.

Também, uma explicação para essa análise diz respeito aos solos da bacia. A sub-bacia em que o ponto T2 captou a coleta de sedimento tem o predomínio de associações de solos como Cambisso-Neossolo e em maior parte Argissolo Bruno-Acinzentado (Miguel et al., 2011). A ausência de Neossolo Flúvico próximo às margens do rio faz com que essas sejam mais estáveis, haja vista as características de instabilidade desses tipos de solos, ainda mais quando formados a partir de materiais com elevado teor de areia.

$\mathrm{O}$ ponto de coleta $\mathrm{T} 3$ apresentou contribuição somente da malha na primeira coleta e de $19 \%$ na

Quadro 4. Resultados do teste de Kruskal-Wallis para cada variável e teor de cada variável no solo das fontes no local de coleta T4 (Coletor 4)

\begin{tabular}{|c|c|c|c|c|c|}
\hline \multirow{2}{*}{ Variável } & \multicolumn{2}{|c|}{ Teste Kruskal-Wallis } & \multicolumn{3}{|c|}{ Concentração média nas fontes } \\
\hline & $H^{(1)}$ & $\mathbf{p}^{(2)}$ & Estrada & Canal & Malha \\
\hline & & & & $-\mathrm{g} \mathrm{kg}^{-1}$ & \\
\hline \multicolumn{6}{|l|}{ Teor } \\
\hline $\mathrm{Al}$ & 8,237 & $0,0163^{*}$ & 27,910 & 23,558 & 19,959 \\
\hline $\mathrm{Fe}$ & 10,164 & $0,0062^{*}$ & 26,816 & 27,814 & 19,873 \\
\hline $\mathrm{Ca}$ & 11,022 & $0,0040^{*}$ & 2,560 & 2,816 & 2,000 \\
\hline $\mathrm{Ba}$ & 11,630 & $0,0030^{*}$ & 0,106 & 0,163 & 0,126 \\
\hline $\mathrm{Mg}$ & 11,939 & $0,0026^{*}$ & 3,913 & 4,184 & 3,295 \\
\hline $\mathrm{La}$ & 11,776 & $0,0028^{*}$ & 0,021 & 0,024 & 0,017 \\
\hline $\mathrm{Li}$ & 9,914 & $0,0070^{*}$ & 0,015 & 0,016 & 0,012 \\
\hline $\mathrm{Mn}$ & 7,181 & $0,0276^{*}$ & 0,657 & 0,918 & 1,049 \\
\hline $\mathrm{Ni}$ & 5,063 & $0,0795^{*}$ & 0,015 & 0,010 & 0,009 \\
\hline $\mathrm{Sr}$ & 26,881 & $0,0000^{*}$ & 0,034 & 0,071 & 0,032 \\
\hline $\mathrm{Zn}$ & 9,435 & $0,0089^{*}$ & 0,019 & 0,026 & 0,020 \\
\hline $\mathrm{K}$ & 0,448 & 0,7992 & 1,871 & 1,715 & 1,982 \\
\hline $\mathrm{Ti}$ & 15,312 & $0,0005^{*}$ & 3,250 & 4,553 & 3,288 \\
\hline $\mathrm{Pb}$ & 16,581 & $0,0003^{*}$ & 0,006 & 0,010 & 0,008 \\
\hline $\mathrm{Cu}$ & 7,298 & $0,0260^{*}$ & 0,050 & 0,049 & 0,034 \\
\hline $\mathrm{Cr}$ & 2,724 & 0,2561 & 0,014 & 0,011 & 0,011 \\
\hline $\mathrm{Na}$ & 16,163 & $0,0003^{*}$ & 0,311 & 0,207 & 0,154 \\
\hline $\mathrm{Be}$ & 15,053 & $0,0005^{*}$ & 0,001 & 0,002 & 0,001 \\
\hline $\mathrm{Co}$ & 4,417 & 0,1099 & 0,019 & 0,022 & 0,020 \\
\hline $\mathrm{P}$ & 9,366 & $0,0093^{*}$ & 0,189 & 0,248 & 0,292 \\
\hline $\mathrm{Si}$ & 1,913 & 0,3842 & 0,135 & 0,133 & 0,123 \\
\hline $\mathrm{V}$ & 14,325 & $0,0008^{*}$ & 0,128 & 0,165 & 0,098 \\
\hline $\mathrm{COT}^{(3)}$ & 30,751 & $0,0000^{*}$ & 8,997 & 17,155 & 18,767 \\
\hline \multicolumn{6}{|c|}{ Dissolução seletiva ${ }^{(4)}$} \\
\hline $\mathrm{Fe}(\mathrm{DCB})$ & 8,956 & $0,0114^{*}$ & 17,650 & 20,300 & 14,367 \\
\hline $\mathrm{Fe}$ (oxa) & 7,294 & $0,0261^{*}$ & 1,597 & 4,335 & 1,676 \\
\hline $\mathrm{Mn}(\mathrm{DCB})$ & 9,916 & $0,0070^{*}$ & 0,462 & 0,719 & 0,742 \\
\hline Mn (oxa) & 2,358 & 0,3076 & 0,273 & 0,415 & 0,343 \\
\hline $\mathrm{Al}(\mathrm{DCB})$ & 1,099 & 0,5771 & 2,801 & 2,249 & 2,206 \\
\hline $\mathrm{Al}$ (oxa) & 1,325 & 0,5154 & 1,589 & 1,220 & 1,455 \\
\hline
\end{tabular}

(1) Valor do teste de discriminação que se aproxima dos valores da distribuição qui-quadrado - $\mathrm{H}_{\text {crítico }}(90 \%$; e 2 gl) $=4,60$; (2) probabilidade de que as fontes diferem entre si, segundo a distribuição qui-quadrado; * $\mathrm{p}<0,10$; ${ }^{(3)} \mathrm{COT}$ : carbono orgânico total e ${ }^{(4)}$ teores de elementos extraídos com Ditionito-citrato-bicarbonato - DCB (Mehra \& Jackson, 1960) e com Oxalato de amônio - oxa (Schwertmann, 1964). 
Quadro 5. Resultado da análise da função discriminante, com acréscimo gradual de cada variável, e os valores correspondentes da minimização do Wilk's Lambda $\left(\Lambda^{*}\right)$

\begin{tabular}{|c|c|c|c|c|c|c|c|c|}
\hline \multirow{2}{*}{ Step } & \multicolumn{2}{|c|}{ T1 } & \multicolumn{2}{|c|}{ T2 } & \multicolumn{2}{|c|}{ T3 } & \multicolumn{2}{|c|}{ T4 } \\
\hline & Variável & $\Lambda^{*(1)}$ & Variável & $\Lambda^{*}$ & Variável & $\Lambda *$ & Variável & $\Lambda^{*}$ \\
\hline 1 & $\mathrm{Fe}$ oxa & 0,182 & COT & 0,522 & $\mathrm{COT}$ & 0,589 & COT & 0,693 \\
\hline 2 & COT & 0,064 & $\mathrm{Sr}$ & 0,278 & $\mathrm{Sr}$ & 0,402 & $\mathrm{Sr}$ & 0,454 \\
\hline 3 & $\mathrm{Al}$ oxa & 0,038 & $\mathrm{Na}$ & 0,201 & $\mathrm{Na}$ & 0,321 & $\mathrm{Na}$ & 0,351 \\
\hline 4 & & & $\mathrm{P}$ & 0,169 & $\mathrm{Fe}$ оха & 0,280 & $\mathrm{Al}$ & 0,285 \\
\hline 5 & & & $\mathrm{Ti}$ & 0,148 & $\mathrm{P}$ & 0,252 & $\mathrm{P}$ & 0,248 \\
\hline 6 & & & & & $\mathrm{Be}$ & 0,221 & $\mathrm{Ti}$ & 0,217 \\
\hline 7 & & & & & Mn oxa & 0,198 & $\mathrm{Ni}$ & 0,196 \\
\hline 8 & & & & & $\mathrm{Ba}$ & 0,183 & $\mathrm{Be}$ & 0,181 \\
\hline 9 & & & & & $\mathrm{Fe}$ & 0,171 & $\mathrm{Fe}$ oxa & 0,167 \\
\hline 10 & & & & & $\mathrm{Ti}$ & 0,154 & $\mathrm{Ba}$ & 0,154 \\
\hline 11 & & & & & Mn DCB & 0,140 & $\mathrm{Mg}$ & 0,145 \\
\hline 12 & & & & & & & $\mathrm{Zn}$ & 0,139 \\
\hline
\end{tabular}

(1) Valores acumulados com a adição de cada variável.

Quadro 6. Fatores de correção para os efeitos da diluição do material grosseiro

\begin{tabular}{|c|c|c|c|}
\hline & Fonte & Areia fina & $100 /\left(100=\mathrm{F}_{\text {ars }}\right)^{(1)}$ \\
\hline & & $\%$ & \\
\hline \multirow[t]{3}{*}{ Coletor 1} & Malha & 13,1 & 1,16 \\
\hline & Canal & 32,0 & 1,55 \\
\hline & Estrada & 16,8 & 1,24 \\
\hline \multirow[t]{3}{*}{ Coletor 2} & Malha & 25,3 & 1,42 \\
\hline & Canal & 27,5 & 1,41 \\
\hline & Estrada & 44,8 & 2,12 \\
\hline \multirow[t]{3}{*}{ Coletor 3} & Malha & 40,1 & 1,83 \\
\hline & Canal & 31,2 & 1,57 \\
\hline & Estrada & 35,5 & 1,64 \\
\hline \multirow[t]{3}{*}{ Coletor 4} & Malha & 51,5 & 1,64 \\
\hline & Canal & 38,2 & 1,53 \\
\hline & Estrada & 35,5 & 1,70 \\
\hline
\end{tabular}

(1) Porcentagem da fração maior que $63 \mu \mathrm{m}$ das amostras das fontes.

segunda, com o aumento da contribuição das estradas numa magnitude de $76 \%$. Esse local engloba a maior quantidade de estradas, comparado aos demais, que drenam a água das chuvas diretamente para as drenagens ou estradas com ausência de pontes, onde há ligação direta entre as estradas e os canais fluviais. $\mathrm{O}$ fato da grande diferença entre uma coleta e outra pode ser explicado por um regime pluviométrico abaixo da média, que ocorreu após a instalação dos coletores, fazendo com que o material que fosse coletado não tivesse tanto a característica da fonte estrada.

As estradas são importante fonte de produção de sedimentos e deve ser dada a devida importância. Em trabalho realizado por Minella et al. (2007), os autores encontraram que as estradas, apesar de ocupar uma área 25 vezes menor que a das lavouras, contribuíram com um terço da produção de sedimentos. Pode ser apontada como causa desse impacto a falta de planejamento da alocação das estradas na bacia (declividade acentuada e grande comprimento), a composição inadequada do material, a baixa frequência de manutenção, a ligação direta entre as estradas e a rede fluvial. Sendo assim, como as estradas representam um componente estático da paisagem agrícola numa bacia hidrográfica, pela sua contribuição na geração de sedimentos, o planejamento de alocação delas é primordial em programas de mitigação de transferência de sedimentos (Collins et al., 2010c).

Por fim, o ponto T4 apresentou contribuição maior da malha (82 \%) na segunda coleta em relação à primeira (77\%) e contribuição menor de estradas (8\%) para a primeira coleta, comparada com a segunda (18\%). À medida que o rio Vacacaí-Mirim se aproxima do reservatório da CORSAN aumentam os problemas relacionados a uso do solo, assoreamento das margens e falta de manutenção de APPs. Apesar da má conservação das margens, a contribuição dos canais se apresentou em caráter pouco significativo. Um fato importante a constar é que o ponto T4 englobou toda a situação da bacia relacionada às fontes de produção de sedimentos, onde a malha provém basicamente de Argissolo Bruno-Acinzentado (Miguel et al., 2011), dando assim a característica de instabilidade relacionada a processos erosivos que ocorrem nesses solos. Isso fica evidente pela formação de erosão em sulcos e até mesmo pelos princípios de voçorocas nessa área. Por mais que o ponto T4, ao contrário dos demais, englobasse as áreas onde são encontrados Neossolo Flúvico, não foi possível identificar a contribuição do canal, exceto para os $15 \%$ da primeira coleta, ficando mais uma vez evidente a instabilidade dos Argissolos Bruno-Acinzentados aos processos de transporte de sedimentos. 

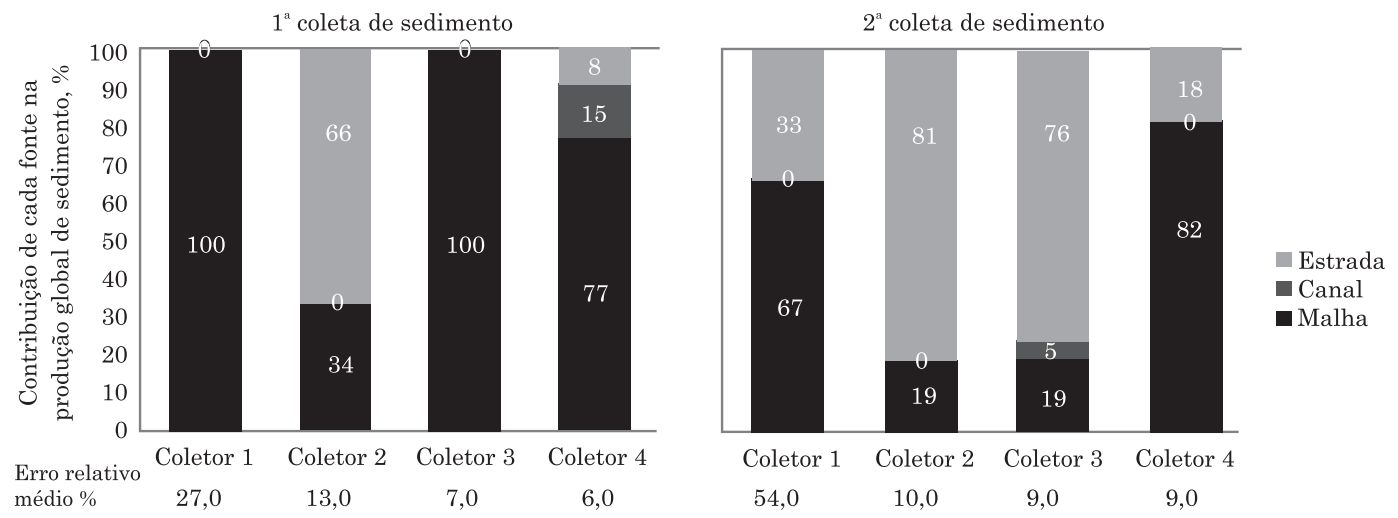

Figura 2. Contribuição relativa das áreas de estrada, canal e malha de amostragem na produção de sedimentos na bacia hidrográfica do rio Vacacaí-Mirim, Santa Maria, RS, para a primeira e segunda coleta de sedimento em suspensão.

\section{CONCLUSÕES}

1. A contribuição de cada uma das fontes de produção de sedimentos (estradas, canais e malha), na bacia hidrográfica do rio Vacacaí-Mirim, foi possível ser identificada, exceto para o ponto T1. O número de elementos químicos determinados na discriminação foi suficiente para serem usados como traçadores. Para o primeiro período de monitoramento, foi identificada uma produção relativa de sedimentos de $60 \%$ das estradas, para o ponto T2; de $100 \%$ da malha, para o T3, e de $77 \%$ da malha, para o T4.

2. As estradas contribuíram relativamente com $81 \%$ do sedimento em suspensão para o ponto T2, com 76 \%, o T3; no T4, a maior contribuição foi da malha com $82 \%$, para o segundo período de monitoramento.

3. As maiores contribuições na produção de sedimentos são provenientes da erosão superficial do solo, onde na primeira coleta de sedimento em suspensão essa predomina nos pontos T3 e T4 e, na segunda coleta, no ponto T4. Sistemas de manejo adequados, manutenção da cobertura do solo e sistemas onde há o menor revolvimento possível do solo têm resultado significativo na diminuição das taxas de produção de sedimentos na área.

4. As estradas apresentam-se como fontes potenciais de erosão onde predominam no ponto T2 para ambas as coletas e, no ponto T3, na segunda coleta. Portanto, a correta alocação e manutenção adequada refletem importante impacto na redução da produção de sedimentos.

\section{LITERATURA CITADA}

BALlantine, D.J.; WALling, D.E.; COLLINS, A.L. \& LEEKS, G.J.L. The content and storage of phosphorus in fine-grained channel bed sediment in contrasting low land agricultural catchments in the UK. Geoderma, 151:141-149, 2009.
BERTOL, I.; ENGEL, F.L.; MAFRA, A.L.; BERTOL, O.B. \& RITTER, S.R. Phosphorus, potassium and organic carbon concentrations in runoff water and sediments under different soil tillage systems during soybean growth. Soil Till. Res., 94:142-150, 2007.

BERTOL, I.; COGO, N.P. \& MIQUELLUTI, D.J. Sedimentos transportados pela enxurrada relacionados à cobertura $\mathrm{e}$ rugosidade superficial do solo e taxa de descarga. Pesq. Agropec. Gaúcha, 3:199-206, 1997.

BERTOL, I.; VIDAL VÁZQUEZ, E.; GONZÁLEZ, A.P.; COGO, N.P.; LUCIANO, R.V. \& FABIAN, E.L. Sedimentos transportados pela enxurrada em eventos de erosão hídrica em um Nitossolo Háplico. R. Bras. Ci. Solo, 34:245$252,2010$.

BIRD, G.; BREWER, P.A.; MACKLIN, M.G.; NIKOLOVA, M.; KOTSEV, T.; MOLLOV, M. \& SWAIN, C. Quantifying sediment-associated metal dispersal using $\mathrm{Pb}$ isotopes: Application of binary and multivariate mixing models at the catchment-scale. Environ. Pollut., 158:2158-2169, 2010.

COGO, N.P.; MOLDENHAUER, W.C. \& FOSTER, G.R. Effect or crop residue, tillage-induced roughness, and runoff velocity on size distribution of eroded soil aggregates. Soil Sci. Soc. Am. J., 47:1005-1008, 1983.

COGO, N.P.; LEVIEN, R. \& SCHWARZ, R.A. Perdas de solo e água por erosão hídrica influenciadas por métodos de preparo, classes de declividade e níveis de fertilidade do solo. R. Bras. Ci. Solo, 27:743-753, 2003.

COLLINS, A.L.; ZHANG, Y.; McCHESNEY, D.; WALLING, D.E.; HALEY, S.M. \& SMITH, P. Sediment source tracing in a lowland agricultural catchment in southern England using a modified procedure combining statistical analysis and numerical modeling. Sci. Total Environ., 414:301$317,2012$.

COLLINS, A.L.; WALLING, D.E.; McMELLIN, G.K.; ZHANG, Y.; GRAY, J.; McGONIGLE, D. \& CHERRINGTON, R. A preliminary investigation of the efûcacy of riparian fencing schemes for reducing contributions from eroding channel banks to the siltation of salmonid spawning gravels across the south west UK. J. Environ. Manage., 91:1341-1349, 2010a. 
COLLINS, A.L.; WALLING, D.E.; WEBB, L. \& KING, P. Apportioning catchment scale sediment sources using a modiûed composite ûngerprinting technique incorporating property weightings and prior information. Geoderma, 155:249-261, 2010b.

COLLINS, A.L.; ZHANG, Y.; WALLING, D.E.; GRENFELL, S.E. \& SMITH, P. Tracing sediment loss from eroding farm tracks using a geochemical ûngerprinting procedure combining local and genetic algorithm optimization. Sci. Total Environ., 408:5461-5471, 2010c.

COLLINS, A.L. \& WALLING, D.E. Selecting fingerprint properties for discriminating tential suspended sediment sources in river basins. J. Hydrol., 261:218244, 2002.

COLLINS, A.L.; WALLING, D.E. \& LEEKS, G.J.L. Source type ascription for fluvial suspended sediment based on a quantitative composite fingerprinting technique. Catena, 29:1-27, 1997.

CUI, Q.; BRANDT, N.; SINHA, R. \& MALMSTRÖM, M.E. Copper content in lake sediments as a tracer of urban emissions: Evaluation through a source-transportstorage model. Sci. Total Environ., 408:2714-2725, 2010.

DU, J.; WU, Y.; HUANG, D. \& ZHANG, J. Use of 7 Be, 210 $\mathrm{Pb}$ and $137 \mathrm{Cs}$ tracers to the transport of surface sediments of the Changjiang Estuary, China. J. Mar. Syst., 82:286-294, 2010.

ENVIRONMENTAL PROTECTION AGENCY - EPA. Method 3050B. Acid digestion of sediments, sludges, and soils, 1996. Disponível em: < http//www.epa.gov/sw846/pdfs/3005A.pdf>. Acesso em 20 mar. 2013.

GONÇALVES, C.S.; RHEINHEIMER, D.S.; PELLEGRINI, J.B.R. \& KIST, S.L. Qualidade da água numa microbacia hidrográfica de cabeceira situada em região produtora de fumo. R. Bras. Eng. Agric. Amb., 9:391$399,2005$.

GUZMÁN, G.; BARRÓN, V. \& GÓMEZ, J.A. Evaluation of magnetic iron oxides as sediment tracers in water erosion experiments. Catena, 82:126-133, 2010.

HE, M.; ZHENG, H.; HUANG, X.; JIA, J. \& LI, L. Yangtze River sediments from source to sink traced with clay mineralogy. J. Asi. Earth Sci., 2012. (article in press). Disponível em: <http://dx.doi.org/10.1016/ j.jseaes.2012.10.001>. Acesso em: 15 mar. 2013.

HOROWITZ, A.J. A primer on sediment trace element chemistry. 2.ed. Chelsea, Lewis, 1991. 136p.

JOHNSON, R.A. \& WICHERN, D.W. Applied multivariate statistical analysis. London, Prentice-Hall International, 1992. $642 \mathrm{p}$.

KIM, W.; DOH, S.J.; YU, Y. \& LEE, Y.I. Magnetic evaluation of sediment provenance in the northern East China Sea using fuzzy c-means cluster analysis. Mar. Geol., 2013. (article in press). Disponível em: $<$ http://dx.doi.org/ 10.1016/j.margeo.2013.01.001>. Acesso em 15 mar. 2013.

MACIEL FILHO, C.L. Carta geotécnica de Santa Maria. Santa Maria, Imprensa Universitária - UFSM, 1990. 21 p.
MARTÍNEZ-CARRERAS, N.; KREIN, A.; GALLART, F.; IFFLY, J.F.; PFISTER, L.; HOFFMANN, L. \& OWENS, P.N. Assessment of different colour parameters for discriminating potential suspended sediment sources and provenance: A multi-scale study in Luxembourg. Geomorphology, 118:118-129, 2010.

MEHRA, O.P. \& JACKSON, M.L. Iron oxide removal from soils and clays by a dithionite-citrate system buffered with sodium bicarbonate. Proc. Clays Clay Miner., 7:317-327, 1960.

MIGUEL, P.; DALMOLIN, R.S.D.; PEDRON, F.A.; SAMUELROSA, A.; MEDEIROS, P.S.C.; MOURA-BUENO, J.M. \& BALBINOT, A. Solo e dinâmica de ocupação das terras em áreas do rebordo do planalto do Rio Grande do Sul. R. Bras. Agroci., 17:447-455, 2011.

MINELLA, J.P.G. \& MERTEN, G.H. Monitoramento de bacias hidrográficas para identificar fontes de sedimentos em suspensão. Ci. Rural, 41:424-432, 2011.

MINELLA, J.P.G.; MERTEN, G.H. \& CLARKE, R.T. Método fingerprinting para identificação de fontes de sedimentos em bacia rural. R. Bras. Eng. Agric. Amb., 13:633-638, 2009a.

MINELLA, J.P.G.; MERTEN, G.H.; WALLING, D.E. \& REICHERT, J.M. Changing sediment yield as an indicator of improved soil management practices in southern Brazil. Catena, 79:228-236, 2009b.

MINELLA, J.P.G.; MERTEN, G.H.; REICHERT, J.M. \& SANTOS, D.R. Identificação e implicações para a conservação do solo das fontes de sedimentos em bacias hidrográficas. R. Bras. Ci. Solo, 31:1637-1646, 2007.

PELLEGRINI, J.B.R.; SANTOS, D.R.; GOLÇALVES, C.S.; COPETTI, A.C.C.; BORTOLUZZI, E.C. \& TESSIER, D. Impacts of anthropic pressures on soil phosphorus availability, concentration, and phosphorus forms in sediments in a Southern Brazilian watershed. J. Soils Sed., 10:451-460, 2010.

SAMUEL-ROSA, A.; MIGUEL, P.; DALMOLIN, R.S.D. \& PEDRON, F.A. Uso da terra no Rebordo do Planalto do Rio Grande do Sul. Ci. Natura, 33:161-173, 2011.

SCHWERTMANN, U. Differenzierung der eisenoxide des bodens durch photochemische extraktion mit saurer ammoniumoxalate. Zeitsch. PflanzenernaehrungDüngund Bodenk, 105:94-202, 1964.

SMALL, I.F.; ROWAN, J.S. \& FRANKS, S.W. Quantitative sediment fingerprinting using a Bayesian uncertainty estimation framework. In: DYER, F.J.; THOMS, M.C. \& OLLEY, J.M., eds. The structure, function, and management implications of fluvial sedimentary. Wallingford, International Association of Hydrological Sciences, 2002. p.443-450.

WALL, G.J. \& WILDING, L.P. Mineralogy and related parameters of fluvial suspended sediments in northwestern Ohio. J. Environ. Qual., 5:168-173, 1976.

WALLING, D.E. \& COLLINS, A.L. Integrated assessment of catchment sediment budgets: A technical manual. Exeter, University of Exeter, 2000. 168p.

WALLING, D.E. Tracing suspended sediment sources in catchments and river systems. Sci. Total Environ., 344:159-184, 2005. 
WALlinG, D.E.; COLLINS, A.L. \& STROUND, RW. Tracing suspended sediment and particulate phosphorus sources in catchments. J. Hydrol., 350:274-289, 2008.

WALLING, D.E. \& WOODWARD, J.C. Tracing sources of suspended sediment in river basins: A case study of the River Culm, Devon, UK. Mar. Freshw. Res., 46:327-336, 1995.
YEOMANS, J.C. \& BREMNER, J.M. A rapid and precise method for routine determination of organic carbon in soil. Commun. Soil Sci. Plant Anal., 19:1467-1476, 1988.

YU, L. \& OLDFIELD, F. A multivariate mixing model for identifying sediment source from magnetic measurements. Quarter. Res., 32:168-181, 1989. 